\title{
Square Difference 3-Equitable Labeling of Paths and Cycles
}

\author{
S. Murugesan, $\mathrm{PhD}$ \\ Department of Mathematics \\ C. B. M. College, Kovaipudur, \\ Coimbatore - 641 042, Tamil Nadu, INDIA
}

\author{
J. Shiama \\ Department of Mathematics, \\ C. M. S. College of Engineering and Technology, \\ Coimbatore- 641 037, Tamil Nadu, INDIA
}

\begin{abstract}
A square difference 3-equitable labeling of a graph $G$ with vertex set $V$ is a bijection $f$ from $V$ to $\{1,2, \ldots,|V|\}$ such that if each edge $u v$ is assigned the label -1 if $\left|[f(u)]^{2}-[f(v)]^{2}\right| \equiv$ $-1(\bmod 4)$, the label 0 if $\left|[f(u)]^{2}-[f(v)]^{2}\right| \equiv 0(\bmod 4)$ and the label 1 if $\left|[f(u)]^{2}-[f(v)]^{2}\right| \equiv 1(\bmod 4)$, then the number of edges labeled with $i$ and the number of edges labeled with $j$ differ by atmost 1 for $-1 \leq i, j \leq 1$. If a graph has a square difference 3 -equitable labeling, then it is called square difference 3-equitable graph. In this paper, we investigate the square difference 3-equitable labeling behaviour of paths and cycles.
\end{abstract}

\section{Keywords}

Square difference 3-equitable labeling, square difference 3equitable graphs

\section{INTRODUCTION}

Definition 1. Let $G=(V, E)$ be a graph. A mapping $f$ : $V(G) \rightarrow\{-1,0,1\}$ is called ternary vertex labeling of $G$ and $f(v)$ is called the label of the vertex $v$ of $G$ under $f$.

For an edge $e=u v$, the induced edge labeling is given by $f^{*}$ : $E(G) \rightarrow\{-1,0,1\}$. Let $v_{f}(-1), v_{f}(0), v_{f}(1)$ be the number of vertices of $G$ having labels $-1,0,1$ respectively under $f$ and $e_{f}(-1), e_{f}(0), e_{f}(1)$ be the number of edges having labels $-1,0$, 1 respectively under $f^{*}$.

DEFINITION 2. A ternary vertex labeling of a graph $G$ is called a 3-equitable labeling if $\left|v_{f}(i)-v_{f}(j)\right| \leq 1$ and $\mid e_{f}(i)-$ $e_{f}(j) \mid \leq 1$ for all $-1 \leq i, j \leq 1$. A graph $G$ is 3 -equitable if it admits 3-equitable labeling.

DEFINITION 3. A square difference 3-equitable labeling of a graph $G$ with vertex set $V(G)$ is a bijection $f: V(G) \rightarrow$ $\{1,2,3, \ldots,|V|\}$ such that the induced edge labeling $f^{*}: E(G) \rightarrow$
$\{-1,0,1\}$ is defined by

$f^{*}(e=u v)=\left\{\begin{aligned}-1 & \text { if }\left|[f(u)]^{2}-[f(v)]^{2}\right| \equiv-1(\bmod 4) \\ 0 & \text { if }\left|[f(u)]^{2}-[f(v)]^{2}\right| \equiv 0(\bmod 4) \\ 1 & \text { if }\left|[f(u)]^{2}-[f(v)]^{2}\right| \equiv 1(\bmod 4)\end{aligned}\right.$

and $\left|e_{f}(i)-e_{f}(j)\right| \leq 1$ for all $-1 \leq i, j \leq 1$. A graph which admits square difference 3-equitable labeling is called square difference 3-equitable graph.

EXAMPLE 1. Consider the following graph $G$.

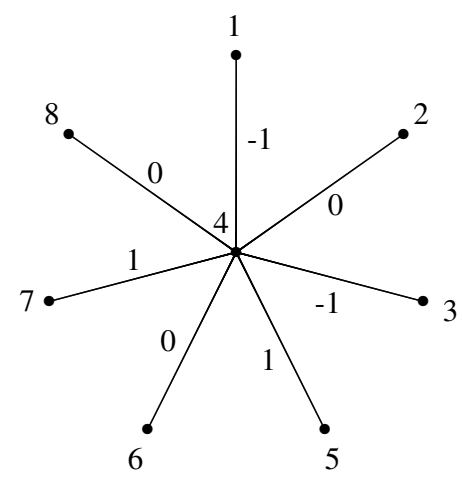

Fig 1. A square difference 3-equitable graph

We see that $e_{f}(-1)=e_{f}(1)=2$ and $e_{f}(0)=3$.

Thus $\left|e_{f}(i)-e_{f}(j)\right| \leq 1$ for all $-1 \leq i, j \leq 1$ and hence $G$ is square difference 3 -equitable.

\section{MAIN RESULTS}

THEOREM 1. The path $P_{n}$ admits square difference 3equitable labeling.

Proof. Let $P_{n}: u_{1} u_{2} \ldots u_{n}$ be the path.

If $n \leq 4$, the following table gives the square difference 3 -equitable labeling of $P_{n}$. 


\begin{tabular}{|c|c|c|c|c|}
\hline$n$ & $u_{1}$ & $u_{2}$ & $u_{3}$ & $u_{4}$ \\
\hline 1 & 1 & & & \\
\hline 2 & 2 & 1 & & \\
\hline 3 & 2 & 3 & 1 & \\
\hline 4 & 2 & 3 & 1 & 4 \\
\hline
\end{tabular}

Table. Square difference 3-equitable labeling of $P_{n}, n \leq 4$ If $n \geq 5$, we consider the following cases.

Case(i): $n \equiv 1(\bmod 6)$

Define

$$
\begin{aligned}
& f\left(u_{1}\right)=2 \\
& f\left(u_{2}\right)=3 \\
& f\left(u_{3}\right)=1 \\
& f\left(u_{4}\right)=4,
\end{aligned}
$$

for $1 \leq i \leq \frac{n-7}{6}$,

$$
\begin{aligned}
f\left(u_{6 i-1}\right) & =6 i+1 \\
f\left(u_{6 i}\right) & =6 i-1 \\
f\left(u_{6 i+1}\right) & =6 i \\
f\left(u_{6 i+2}\right) & =6 i+2 \\
f\left(u_{6 i+3}\right) & =6 i+3 \\
f\left(u_{6 i+4}\right) & =6 i+4
\end{aligned}
$$

and

$$
\begin{aligned}
f\left(u_{n-2}\right) & =n \\
f\left(u_{n-1}\right) & =n-2 \\
f\left(u_{n}\right) & =n-1 .
\end{aligned}
$$

Then

$$
\begin{gathered}
\left|\left[f\left(u_{1}\right)\right]^{2}-\left[f\left(u_{2}\right)\right]^{2}\right| \equiv 1(\bmod 4) \\
\Rightarrow f^{*}\left(u_{1} u_{2}\right)=1 \\
\left|\left[f\left(u_{2}\right)\right]^{2}-\left[f\left(u_{3}\right)\right]^{2}\right| \equiv 0(\bmod 4) \\
\Rightarrow f^{*}\left(u_{2} u_{3}\right)=0 \\
\left|\left[f\left(u_{3}\right)\right]^{2}-\left[f\left(u_{4}\right)\right]^{2}\right| \equiv-1(\bmod 4) \\
\Rightarrow f^{*}\left(u_{3} u_{4}\right)=-1,
\end{gathered}
$$

for $1 \leq i \leq \frac{n-7}{6}$,

$$
\begin{aligned}
\left|\left[f\left(u_{6 i-2}\right)\right]^{2}-\left[f\left(u_{6 i-1}\right)\right]^{2}\right| & \equiv 1(\bmod 4) \\
\Rightarrow f^{*}\left(u_{6 i-2} u_{6 i-1}\right) & =1 \\
\left|\left[f\left(u_{6 i-1}\right)\right]^{2}-\left[f\left(u_{6 i}\right)\right]^{2}\right| & \equiv 0(\bmod 4) \\
\Rightarrow f^{*}\left(u_{6 i-1} u_{6 i}\right) & =0 \\
\left|\left[f\left(u_{6 i}\right)\right]^{2}-\left[f\left(u_{6 i+1}\right)\right]^{2}\right| & \equiv-1(\bmod 4) \\
\Rightarrow f^{*}\left(u_{6 i} u_{6 i+1}\right) & =-1 \\
\mid\left[f\left(u_{6 i+1}\right)\right]^{2}-\left[f\left(u_{6 i+2}\right)\right]^{2} & \equiv 0(\bmod 4) \\
\Rightarrow f^{*}\left(u_{6 i+1} u_{6 i+2}\right) & =0 \\
\mid\left[f\left(u_{6 i+2}\right)\right]^{2}-\left[f\left(u_{6 i+3}\right)\right]^{2} & \equiv 1(\bmod 4) \\
\Rightarrow f^{*}\left(u_{6 i+2} u_{6 i+3}\right) & =1 \\
\left|\left[f\left(u_{6 i+3}\right)\right]^{2}-\left[f\left(u_{6 i+4}\right)\right]^{2}\right| & \equiv-1(\bmod 4) \\
\Rightarrow f^{*}\left(u_{6 i+3} u_{6 i+4}\right) & =-1
\end{aligned}
$$

and

$$
\begin{gathered}
\left|\left[f\left(u_{n-3}\right)\right]^{2}-\left[f\left(u_{n-2}\right)\right]^{2}\right| \equiv 1(\bmod 4) \\
\Rightarrow f^{*}\left(u_{n-3} u_{n-2}\right)=1 \\
\left|\left[f\left(u_{n-2}\right)\right]^{2}-\left[f\left(u_{n-1}\right)\right]^{2}\right| \equiv 0(\bmod 4) \\
\Rightarrow f^{*}\left(u_{n-2} u_{n-1}\right)=0 \\
\left|\left[f\left(u_{n-1}\right)\right]^{2}-\left[f\left(u_{n}\right)\right]^{2}\right| \equiv-1(\bmod 4) \\
\Rightarrow f^{*}\left(u_{n-1} u_{n}\right)=-1 .
\end{gathered}
$$

Thus $e_{f}(-1)=e_{f}(0)=e_{f}(1)=\frac{n-1}{3}$.

Case(ii): $n \equiv 2(\bmod 6)$

Define

$$
\begin{aligned}
& f\left(u_{1}\right)=2 \\
& f\left(u_{2}\right)=3 \\
& f\left(u_{3}\right)=1 \\
& f\left(u_{4}\right)=4,
\end{aligned}
$$

for $1 \leq i \leq \frac{n-8}{6}$,

$$
\begin{aligned}
f\left(u_{6 i-1}\right) & =6 i+1 \\
f\left(u_{6 i}\right) & =6 i-1 \\
f\left(u_{6 i+1}\right) & =6 i \\
f\left(u_{6 i+2}\right) & =6 i+2 \\
f\left(u_{6 i+3}\right) & =6 i+3 \\
f\left(u_{6 i+4}\right) & =6 i+4
\end{aligned}
$$

and

$$
\begin{aligned}
f\left(u_{n-3}\right) & =n-1 \\
f\left(u_{n-2}\right) & =n-3 \\
f\left(u_{n-1}\right) & =n-2 \\
f\left(u_{n}\right) & =n .
\end{aligned}
$$

Then $e_{f}(-1)=e_{f}(1)=\frac{n-2}{3}$ and $e_{f}(0)=\frac{n+1}{3}$.

Case(iii): $n \equiv 3(\bmod 6)$

Define

$$
\begin{aligned}
& f\left(u_{1}\right)=2 \\
& f\left(u_{2}\right)=3 \\
& f\left(u_{3}\right)=1 \\
& f\left(u_{4}\right)=4,
\end{aligned}
$$

for $1 \leq i \leq \frac{n-9}{6}$, 
and

$$
\begin{aligned}
f\left(u_{n-4}\right) & =n-2 \\
f\left(u_{n-3}\right) & =n-4 \\
f\left(u_{n-2}\right) & =n-3 \\
f\left(u_{n-1}\right) & =n-1 \\
f\left(u_{n}\right) & =n .
\end{aligned}
$$

Then $e_{f}(-1)=\frac{n-3}{3}$ and $e_{f}(0)=e_{f}(1)=\frac{n}{3}$.

Case(iv): $n \equiv 4(\bmod 6)$

Define

$$
\begin{aligned}
& f\left(u_{1}\right)=2 \\
& f\left(u_{2}\right)=3 \\
& f\left(u_{3}\right)=1 \\
& f\left(u_{4}\right)=4
\end{aligned}
$$

and for $1 \leq i \leq \frac{n-4}{6}$,

$$
\begin{aligned}
f\left(u_{6 i-1}\right) & =6 i+1 \\
f\left(u_{6 i}\right) & =6 i-1 \\
f\left(u_{6 i+1}\right) & =6 i \\
f\left(u_{6 i+2}\right) & =6 i+2 \\
f\left(u_{6 i+3}\right) & =6 i+3 \\
f\left(u_{6 i+4}\right) & =6 i+4 .
\end{aligned}
$$

Then $e_{f}(-1)=e_{f}(0)=e_{f}(1)=\frac{n-1}{3}$.

Case $(\mathrm{v}): n \equiv 5(\bmod 6)$

Define

$$
\begin{aligned}
& f\left(u_{1}\right)=2 \\
& f\left(u_{2}\right)=3 \\
& f\left(u_{3}\right)=1 \\
& f\left(u_{4}\right)=4,
\end{aligned}
$$

for $1 \leq i \leq \frac{n-5}{6}$,

$$
\begin{aligned}
f\left(u_{6 i-1}\right) & =6 i+1 \\
f\left(u_{6 i}\right) & =6 i-1 \\
f\left(u_{6 i+1}\right) & =6 i \\
f\left(u_{6 i+2}\right) & =6 i+2 \\
f\left(u_{6 i+3}\right) & =6 i+3 \\
f\left(u_{6 i+4}\right) & =6 i+4
\end{aligned}
$$

and

$$
f\left(u_{n}\right)=n .
$$

Then $e_{f}(-1)=e_{f}(0)=\frac{n-2}{3}$ and $e_{f}(1)=\frac{n+1}{3}$.

Case(vi): $n \equiv 0(\bmod 6)$

Define

$$
\begin{aligned}
& f\left(u_{1}\right)=2 \\
& f\left(u_{2}\right)=3 \\
& f\left(u_{3}\right)=1 \\
& f\left(u_{4}\right)=4,
\end{aligned}
$$

for $1 \leq i \leq \frac{n-6}{6}$,

$$
\begin{aligned}
f\left(u_{6 i-1}\right) & =6 i+1 \\
f\left(u_{6 i}\right) & =6 i-1 \\
f\left(u_{6 i+1}\right) & =6 i \\
f\left(u_{6 i+2}\right) & =6 i+2 \\
f\left(u_{6 i+3}\right) & =6 i+3 \\
f\left(u_{6 i+4}\right) & =6 i+4
\end{aligned}
$$

and

$$
\begin{aligned}
f\left(u_{n-1}\right) & =n-1 \\
f\left(u_{n}\right) & =n .
\end{aligned}
$$

Then $e_{f}(-1)=e_{f}(1)=\frac{n}{3}$ and $e_{f}(0)=\frac{n-3}{3}$.

Thus in all cases, $\left|e_{f}(i)-e_{f}(j)\right| \leq 1$ for all $-1 \leq i, j \leq 1$ and therefore $P_{n}$ is a square difference 3-equitable graph.

EXAMPLE 2. The square difference 3-equitable labeling of $P_{10}$ is shown below.

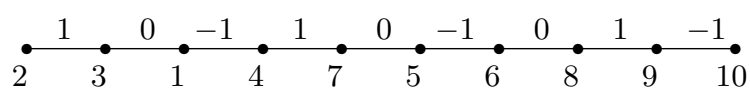

Fig 2. Square difference 3-equitable labeling of $P_{10}$

THEOREM 2. The cycle $C_{n}$ admits square difference 3equitable labeling.

Proof. Let $C_{n}: u_{1} u_{2} \ldots u_{n} u_{1}$ be the cycle.

The square difference 3-equitable labeling of $C_{3}$ is given as follows.

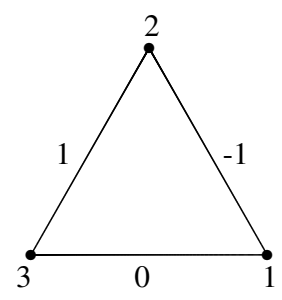

Fig 3. Square difference 3-equitable labeling of $C_{3}$

If $n \geq 4$, we consider the following cases.

Case $(\mathrm{i}): n \equiv 1(\bmod 6)$

Define

$$
\begin{aligned}
& f\left(u_{1}\right)=2 \\
& f\left(u_{2}\right)=3 \\
& f\left(u_{3}\right)=1 \\
& f\left(u_{4}\right)=4,
\end{aligned}
$$


for $1 \leq i \leq \frac{n-7}{6}$,

$$
\begin{aligned}
f\left(u_{6 i-1}\right) & =6 i+1 \\
f\left(u_{6 i}\right) & =6 i-1 \\
f\left(u_{6 i+1}\right) & =6 i \\
f\left(u_{6 i+2}\right) & =6 i+2 \\
f\left(u_{6 i+3}\right) & =6 i+3 \\
f\left(u_{6 i+4}\right) & =6 i+4
\end{aligned}
$$

and

$$
\begin{aligned}
f\left(u_{n-2}\right) & =n \\
f\left(u_{n-1}\right) & =n-2 \\
f\left(u_{n}\right) & =n-1 .
\end{aligned}
$$

Then $e_{f}(-1)=e_{f}(1)=\frac{n-1}{3}$ and $e_{f}(0)=\frac{n+2}{3}$.

Case(ii): $n \equiv 2(\bmod 6)$

Define

$$
\begin{aligned}
& f\left(u_{1}\right)=2 \\
& f\left(u_{2}\right)=1 \\
& f\left(u_{3}\right)=4 \\
& f\left(u_{4}\right)=5 \\
& f\left(u_{5}\right)=3,
\end{aligned}
$$

for $1 \leq i \leq \frac{n-8}{6}$,

$$
\begin{aligned}
f\left(u_{6 i}\right) & =6 i+2 \\
f\left(u_{6 i+1}\right) & =6 i \\
f\left(u_{6 i+2}\right) & =6 i+1 \\
f\left(u_{6 i+3}\right) & =6 i+3 \\
f\left(u_{6 i+4}\right) & =6 i+4 \\
f\left(u_{6 i+5}\right) & =6 i+5
\end{aligned}
$$

and

$$
\begin{aligned}
f\left(u_{n-2}\right) & =n \\
f\left(u_{n-1}\right) & =n-2 \\
f\left(u_{n}\right) & =n-1 .
\end{aligned}
$$

Then $e_{f}(-1)=e_{f}(1)=\frac{n+1}{3}$ and $e_{f}(0)=\frac{n-2}{3}$.

Case(iii): $n \equiv 3(\bmod 6)$

Define

$$
\begin{aligned}
& f\left(u_{1}\right)=2 \\
& f\left(u_{2}\right)=3 \\
& f\left(u_{3}\right)=5 \\
& f\left(u_{4}\right)=4 \\
& f\left(u_{5}\right)=1 \\
& f\left(u_{6}\right)=6,
\end{aligned}
$$

for $1 \leq i \leq \frac{n-9}{6}$,

$$
\begin{aligned}
& f\left(u_{6 i+1}\right)=6 i+3 \\
& f\left(u_{6 i+2}\right)=6 i+1 \\
& f\left(u_{6 i+3}\right)=6 i+2 \\
& f\left(u_{6 i+4}\right)=6 i+4 \\
& f\left(u_{6 i+5}\right)=6 i+5 \\
& f\left(u_{6 i+6}\right)=6 i+6
\end{aligned}
$$

and

$$
\begin{aligned}
f\left(u_{n-2}\right) & =n \\
f\left(u_{n-1}\right) & =n-2 \\
f\left(u_{n}\right) & =n-1 .
\end{aligned}
$$

Then $e_{f}(-1)=e_{f}(0)=e_{f}(1)=\frac{n}{3}$.

Case(iv): $n \equiv 4(\bmod 6)$

Define

$$
\begin{aligned}
& f\left(u_{1}\right)=2 \\
& f\left(u_{2}\right)=3 \\
& f\left(u_{3}\right)=1 \\
& f\left(u_{4}\right)=4
\end{aligned}
$$

and for $1 \leq i \leq \frac{n-4}{6}$,

$$
\begin{aligned}
f\left(u_{6 i-1}\right) & =6 i+1 \\
f\left(u_{6 i}\right) & =6 i-1 \\
f\left(u_{6 i+1}\right) & =6 i \\
f\left(u_{6 i+2}\right) & =6 i+2 \\
f\left(u_{6 i+3}\right) & =6 i+3 \\
f\left(u_{6 i+4}\right) & =6 i+4 .
\end{aligned}
$$

Then $e_{f}(-1)=e_{f}(1)=\frac{n-1}{3}$ and $e_{f}(0)=\frac{n+2}{3}$.

Case $(\mathrm{v}): n \equiv 5(\bmod 6)$

Define

$$
\begin{aligned}
& f\left(u_{1}\right)=2 \\
& f\left(u_{2}\right)=1 \\
& f\left(u_{3}\right)=4 \\
& f\left(u_{4}\right)=5 \\
& f\left(u_{5}\right)=3
\end{aligned}
$$

and for $1 \leq i \leq \frac{n-5}{6}$,

$$
\begin{aligned}
f\left(u_{6 i}\right) & =6 i+2 \\
f\left(u_{6 i+1}\right) & =6 i \\
f\left(u_{6 i+2}\right) & =6 i+1 \\
f\left(u_{6 i+3}\right) & =6 i+3 \\
f\left(u_{6 i+4}\right) & =6 i+4 \\
f\left(u_{6 i+5}\right) & =6 i+5 .
\end{aligned}
$$

Then $e_{f}(-1)=e_{f}(1)=\frac{n+1}{3}$ and $e_{f}(0)=\frac{n-2}{3}$. 
Case(vi): $n \equiv 0(\bmod 6)$

Define

$$
\begin{aligned}
& f\left(u_{1}\right)=2 \\
& f\left(u_{2}\right)=3 \\
& f\left(u_{3}\right)=5 \\
& f\left(u_{4}\right)=4 \\
& f\left(u_{5}\right)=1 \\
& f\left(u_{6}\right)=6
\end{aligned}
$$

and for $1 \leq i \leq \frac{n-6}{6}$,

$$
\begin{aligned}
& f\left(u_{6 i+1}\right)=6 i+3 \\
& f\left(u_{6 i+2}\right)=6 i+1 \\
& f\left(u_{6 i+3}\right)=6 i+2 \\
& f\left(u_{6 i+4}\right)=6 i+4 \\
& f\left(u_{6 i+5}\right)=6 i+5 \\
& f\left(u_{6 i+6}\right)=6 i+6 .
\end{aligned}
$$

Then $e_{f}(-1)=e_{f}(0)=e_{f}(1)=\frac{n}{3}$.

Thus in all cases, $\left|e_{f}(i)-e_{f}(j)\right| \leq 1$ for all $-1 \leq i, j \leq 1$ and therefore $C_{n}$ is a square difference 3-equitable graph.

EXAMPLE 3. The square difference 3-equitable labeling of $\mathrm{C}_{9}$ is shown below.

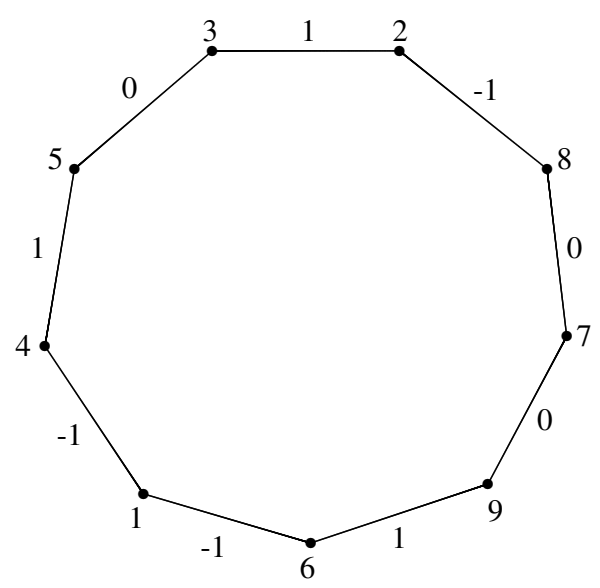

Fig 4. Square difference 3-equitable labeling of $C_{9}$

\section{CONCLUSION}

We have discussed here a new labeling called square difference 3equitable labeling and we have investigated for paths and cycles only. The results reported here are new and expected to add new dimension to the theory of 3- equitable graphs. It is possible to investigate similar results for other graph families.

\section{REFERENCES}

[1] F. Harary, Graph Theory, Addition-Wesley, Reading, Mass, 1972.
[2] J. A. Gallian, A dynamic survey of graph labeling, Electronic Journal of Combinatorics, 17 (2010), DS6.

[3] J.Shiama, Square sum labeling for some middle and total graphs, International Journal of Computer Applications (0975-8887) Volume 37- No.4 January 2012.

[4] J.Shiama, Square difference labeling for some graphs, International Journal of Computer Applications (0975-8887) Volume 44- No.4, April 2012.

[5] J.Shiama, Some Special types of Square difference graphs, International Journal of Mathematical archives- 3(6), 2012, 2369-2374 ISSN 2229-5046.

[6] J.Shiama, Square difference labeling for some path, fan and gear graphs, International Journal of Scientific and Engineering Research volume 4, issues 3, March -2013, ISSN 22295518 . 